\title{
ADAPTIVE BONE-REMODELING THEORY APPLIED TO PROSTHETIC-DESIGN ANALYSIS*
}

\author{
R. Huiskes, H. Weinans, H. J. Grootenboert, M. Dalstra, B. Fudala and \\ T. J. SLOOFF \\ Biomechanics Section, Institute of Orthopaedics, University of Nijmegen, $6500 \mathrm{HB}$ Nijmegen. \\ The Netherlands; +University of Twente. Enschede, The Netherlands
}

\begin{abstract}
The subject of this article is the development and application of computer-simulation methods to predict stress-related adaptive bone remodeling, in accordance with 'Wolf s Law'. These models are based on the Finite Element Method (FEM) in combination with numerical formulations of adaptive boneremodeling theories.

In the adaptive remodeling models presented, the Strain Energy Density (SED) is used as a feed-back control variable to determine shape or bone density adaptations to alternative functional requirements. whereby homeostatic SED distribution is assumed as the remodeling objective.

These models are applied to investigate the relation between 'stress shielding' and bone resorption in the femoral cortex around intramedullary prostheses, such as used in Total Hip Arthroplasty (THA). It is shown that the amount of bone resorption depends mainly on the rigidity and the bonding characteristics of the implant. Homeostatic SED can be obtained when the resorption process occurs at the periosteal surface. rather than inside the cortex, provided that the stem is adequately flexible.
\end{abstract}

\section{INTRODUCTION}

One effect of total joint replacement is that it changes the normal stress patterns in the bones. As a result, the bone may engage in a process of remodeling, whereby the local bone mass is adapted to the new functional requirements. Such a process could endanger the longterm success of the replacement since, obviously, a prosthetic component will not remain well-fixed for long when its bone bed gradually disappears. On the other hand, remodeling may also have a beneficial effect, when it strengthens the bone/prosthesis composite as a whole. Evidently, orthopaedic surgery would be greatly served if the adaptive changes around prostheses could, somehow, be predicted, if only by approximation. The development of analytical methods for this purpose is the subject of this article.

The idea that bone adaptations around prostheses, as they are indeed seen in the orthopaedic clinic, are effects of abnormal bone stresses, is derived from 'Wolff's Law', stating, generally speaking, that a relation exists between stress transfer and architecture in bones, and that bone is able to adapt its structure to the actual stress patterns. Or, in other words, that bone, in the first place, is an optimal structure relative to its mechanical requirements and, secondly, that bone is able to maintain an optimal configuration relative to alternative mechanical requirements. Alternative requirements may be caused by changes. in

Editor's note. Submitted by invitation for the special 'F. Gaynor Evans Anniversary Issue on Bone Biomechanics: (J. D. Currey, D. R. Carter, A. Viidik, Guest Editors.)

* Presented in part at the Fifth General Meeting of the European Society of Biomechanics, Berlin, F.R.G., September 1986. loading, but also by prosthetic replacements. The stress patterns in bone/prosthesis structures depend on the external loading conditions, but also on the geometry and material properties of the bone and the prosthesis. Given adequate quantitative descriptions of loading, geometry, material properties and boundary conditions in the structure, the degree of bone-stress abnormality relative to the intact bone can be predicted using finite element analysis (Huiskes and Chao, 1983). What is left to be determined, then, is the adaptive remodeling process based on these predicted stress abnormalities. To accomplish that, some sort of quantitative adaptive remodeling theory is needed. 'Wolff's Law' itself, as it is commonly understood, is not a scientific law in the traditional sense, but rather a series of qualitative observations and expectations relative to bone architecture and stress transfer, blended with the theory of functional adaptation developed by Roux (Roesler, 1981). Hence, it is not a theory suitable for quantitative predictions; the law is in fact not falsifiable. Several attempts were made to augment or replace 'Wolff's Law' by a more rigorous mathematical description (Koch, 1917; Pauwels, 1965; Frost, 1964; Kummer, 1972; Cowin and Hegedus, 1976; Hayes and Snyder, 1981; Fyhrie and Carter, 1986a; Cowin, 1986). Theories of this kind can be applied in computer-simulation procedures, in combination with the Finite Element Method (FEM), to be used for predictions of adaptive bone remodeling around prostheses.

The purpose of this article is the presentation of such adaptive-remodeling simulation models, a discussion of their applicability and their relation to similar models, and their application to the problem of 'stress shielding' and adaptive cortical-bone remodeling 
around intramedullary prostheses. such as femoral stems in Total Hip Arthroplasty (THA).

\section{A COMPLTER SIMLLATION MODEL FOR ADAPTIVE BONE REMODELING}

\section{Bone-remodeling theories}

Of the existing bone-remodeling theories, two will be described in some detail. They have their empirical nature in common, by relating stress or strain in bone to adaptive remodeling behavior, without considering the actual biological mechanisms governing the relationship. They also have in common that bone is considered as a continuum material, for which the theories of linear elasticity are valid. Finally, the theories are (quasi) static ones; effects of loading rates, visco-elastic effects and inertial effects are not considered.

Wolff (1869) considered cortical bone as 'densified' trabecular bone, so that these two tissues were basically the same, differing only in their porosities. Fyhrie and Carter (1986a) took the same approach, when developing a unifying theory to predict the density and trabecular architecture of bone. They assume bone tissue to be a self-optimizing material with the objective of aligning trabecular architecture with principalstress orientation, and adapting its apparent density $\rho$ (Carter and Hayes, 1977) to an "effective stress" $\sigma_{\text {efs }}$. Based on strength or strain-optimization criteria, they derive

$$
\rho=A \sigma_{\text {ef }}^{\mathrm{x}},
$$

whereby $A$ and $\alpha$ are constants. The effective stress is determined from either a failure or an elastic energy criterion. This unified theory can indeed be used to quantitatively predict bone architecture and adaptive remodeling, and is also falsifiable in principle, although rigorous attempts in this direction have not been reported. Nevertheless, the theory is compatible with 'Wolff's Law' and also with combined analytical/experimental findings such as reported by Hayes and Snyder (1981).

In later publications (Carter et al., 1987a, b; Fyhrie and Carter, 1986b) they emphasize the application of the theory to predict the optimal density distribution of the proximal femur according to the above criterion, using a numerical formulation in combination with the FEM. They assume $\alpha=1 / 2$ and the effective stress to be derived from the apparent strain energy density ( $\mathrm{SED}=$ strain energy/bone volume), according to $\sigma_{e f S}^{2}=2 E U$, where $E$ is the 'average' apparent elastic modulus, and $U$ the apparent strain energy density (SED). Also applying the modulus/density relation $E$ $=c \rho^{3}$, where $c$ is a constant (Carter and Hayes, 1977), their optimization function in fact transforms to

$$
\rho=c^{\prime} U,
$$

where $c^{\prime}$ is a constant. It is noteworthy that their optimization function can also be written in the form
$U_{b}=$ constant, where $U_{b}$ is the "true' or bulk SED in the bone (trabeculae) itself, since $U=\rho U_{b} / \rho_{c}$, provided that the cortical bone density, $\rho_{c}$, equals the 'true' or bulk density of the bone (trabeculae) itself.

The above formulation is only one of the possible ones in which the "self optimization' theory of Fyhrie and Carter (1986a) can be applied. This part, of course, only concerns the bone density, whereas the unifying theory as a whole also involves trabecular orientation.

The theory of 'adaptive elasticity' (Cowin and Hegedus, 1976; Hegedus and Cowin, 1976; Cowin and Firoozbakhsh, 1981) was developed to describe the remodeling behavior of cortical bone. This theory primarily attempts to describe the adaptive remodeling behavior of bone from one loading configuration to another, rather than predicting the optimal structure of normal bone, as in the theory of self-optimization described above. For this purpose it is assumed that cortical bone tissue has a site-specific natural or homeostatic equilibrium strain state. A change of load or, in fact, an abnormal actual strain state will stimulate the bone tissue to adapt its mass in such a way, that the equilibrium strain state is again obtained. In the theory, the rate of adaptation is coupled to the difference between the equilibrium and the actual strain states.

Following a suggestion by Frost (1964), Cowin and associates separate internal and surface (or external) remodeling. In the first case, the bone has only the option of adapting its density, thereby, assuming continuum theory to be valid, adapting its elastic modulus according to

$$
\frac{\mathrm{d} E}{\mathrm{~d} t}=A_{i j}\left(\varepsilon_{i j}-\varepsilon_{i j}^{0}\right),
$$

where $E$ is the local modulus of elasticity, $\varepsilon_{i j}$ the actual strain tensor, $\varepsilon_{i j}^{0}$ the equilibrium strain tensor, and $A_{i j}$ a matrix of remodeling coefficients.

In the case of external remodeling the bone can only add or remove material on the periosteal and endosteal surfaces, stimulated by the strain state at those surfaces, according to

$$
\frac{\mathrm{d} X}{\mathrm{~d} t}=B_{i j}\left(c_{i j}-\varepsilon_{i j}^{0}\right),
$$

where $X$ is a characteristic surface coordinate perpendicular to the surface, and $B_{i j}$ again a matrix of remodeling coefficients.

Cowin and associates also considered quadratic relations between strain and rate of adaptation (Firoozbakhsh and Cowin, 1981), and performed a number of studies to determine possible values of the remodeling coefficients. Recently, using the theory of external remodeling to simulate animal experiments reported in the literature, they found quite reasonable agreement between experimental results and theoretical predictions (Cowin et al., 1985). The theory of adaptive elasticity was used by Hart et al. (1984) in relation with a three-dimensional computational model based on the FEM. 


\section{A computer simulation model}

The computer model developed in our group is based on the use of the two-dimensional Finite Element Method (FEM) in combination with an alternative formulation of the theory of adaptive elasticity. As the first difference, not the strain tensor is used as a feed-back control variable, but the strain energy density (SED)

$$
U=\frac{1}{2} \varepsilon_{i j} \sigma_{i j}
$$

where $\sigma_{i j}$ is the local stress tensor and $\varepsilon_{i j}$ the strain tensor. The difference between the actual SED, $U$, and a site-specific homeostatic equilibrium SED, $U_{n}$, is assumed as the driving force for adaptive activity.

In the second place, instead of assuming a linear relation between adaptive rate and SED, bone is assumed to be 'lazy', following a suggestion from Carter (1984). In effect, it is assumed that a certain threshold level in over- or underloading must be exceeded before bone reacts. This is illustrated in Fig. 1. The local actual SED, $U(t, x)$, is referred to an homeostatic SED, $U_{n}(x)$, where $t$ is the time and $x$ the location vector. When $U>(1+s) U_{n}$ or $U<(1-s) U_{n}$. adaptive activity is initiated, whereby $s$ is the threshold level, expressed as a fraction of $U_{n}$, and the rate of adaptation is proportional to $\mathrm{C}$, the slope of the curves in Fig. 1.

Hence, for internal remodeling

$$
\begin{aligned}
& \frac{\mathrm{d} E}{\mathrm{~d} t}= \\
& \left.\qquad \begin{array}{lr}
C_{e}\left(U-(1+s) U_{n}\right), & U>(1+s) U_{n} \\
0, & (1-s) U_{n} \leqslant U \leqslant(1+s) U_{n} \\
C_{e}\left(U-(1-s) U_{n}\right), & U<(1-s) U_{n}
\end{array}\right]
\end{aligned}
$$

where $E$ is the elastic modulus in the point concerned. A similar formula is used for external remodeling, whereby $\mathrm{d} X / \mathrm{d} t$, the rate of surface growth perpendicular to the surface, is expressed in the same form as in equation (6).

In the case that $s=0$, we have, for internal

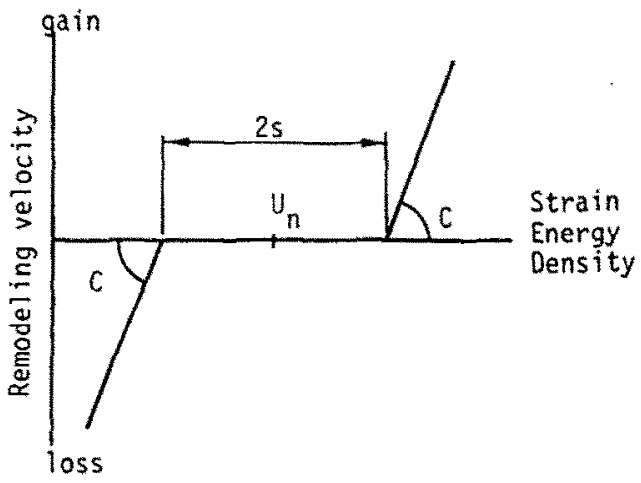

Fig. 1. The assumed, trilinear adaptive remodeling rate as a function of the SED. remodeling

$$
\frac{d E}{d t}=C_{e}\left(U-U_{n}\right)
$$

and for external remodeling

$$
\frac{d X}{d t}=C_{x}\left(U-U_{n}\right)
$$

Computer programs to evaluate the changes in modulus and geometry, based on a change in SED, were developed and combined with the FEM codes NONSYS and MARC (Marc Analysis Corporation, Palo Alto, CA), as illustrated in Fig. 2. To determine the geometrical or material changes, the remodeling rates are integrated, using forward Euler integration with a constant time step $\Delta t$, whereby, for internal remodeling, the change in elastic modulus in each step follows from $(s=0)$

$$
\begin{aligned}
\Delta E^{i}=E^{i}(t+\Delta t)-E^{i}(t)=\Delta t C_{e}\left\{U^{i}(t)-U_{n}^{i}\right\} & \\
1 & \leqslant i \leqslant n,
\end{aligned}
$$

where $n$ is the number of elements. For external remodeling the relocations of the surface points perpendicular to the surface in each step follow from $(s=0)$

$$
\begin{array}{r}
\Delta X^{j}=X^{j}(t+\Delta t)-X^{j}(t)=\Delta t C_{x}\left\{U^{j}(t)-U_{n}^{j}\right\}, \\
1 \leqslant j \leqslant m,
\end{array}
$$

where $m$ is the number of surface nodal points concerned. If $s \neq 0$, similar formulas are applied, corresponding to equation (6).

The constants $C_{e}$ and $C_{x}$ determine the remodeling rate. Since values for these constants are, as yet, unknown, they were given arbitrary values. Hence, only the final result of the remodeling process is considered as realistic. However, to insure stability of the iteration process, the time step must be adequately small. This problem is solved empirically by selecting values for $\Delta t C_{p}$ and $\Delta t C_{x}$ after a trial run for each specific application, ensuring that $\Delta E$ (or $\Delta X$ ) in the first iterative step is small relative to its actual value $(10-20 \%)$ at the location where $\left|U-U_{n}\right|$ has the highest value. In this case monotonic (approximately exponential) convergence occurs in most cases. It must be noted, however, that convergence is problemdependent and not always automatically insured.

The elements presently applied in the FEM code are two-dimensional plane-strain quadrilaterals with a quadratic displacement field ( 8 nodal points), but this is not a principle restriction. In the case of internal remodeling, the SED is evaluated per element, averaging the values in the element integration points. In the case of external remodeling the SED is evaluated in the surface nodal points. Problems occur when the elastic modulus becomes negative or when an element shape deteriorates due to excessive nodal-point relocations. To avoid that, ad hoc precautions are taken in each specific application, i.e. relocations of other than 


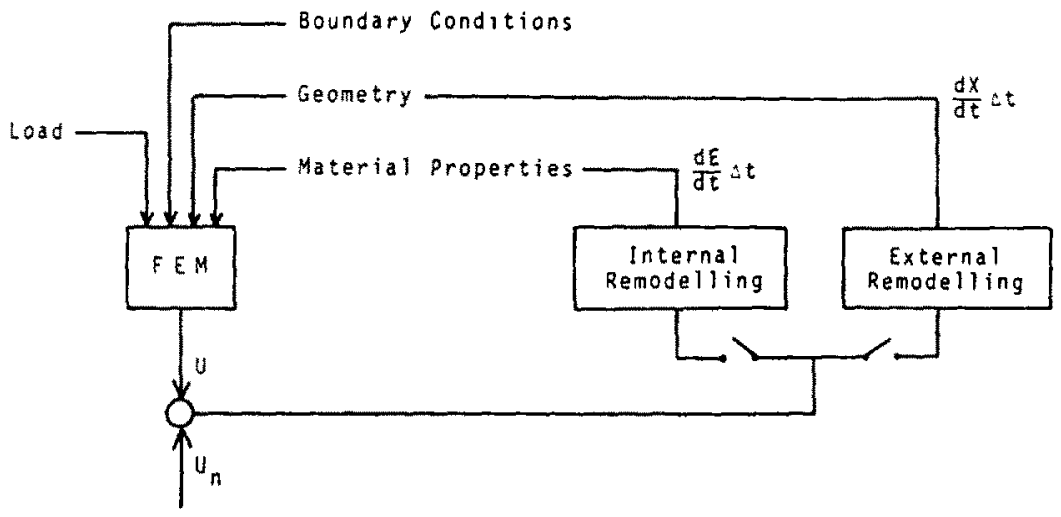

Fig. 2. Schematic representation of the adaptive remodeling program, integrated with the finite element method (FEM) code. The feed-back control variable is the difference between the actual SED, $U$, and the homeostatic SED, $U_{n}$.

surface nodal points, the use of lower boundaries for the elastic moduli, and lower boundaries for the element thickness. The physical interpretation of the latter precautions is, that an extremely flexible element can, for all practical purposes, be considered as effectively removed.

\section{Application to adaptive shape optimization}

Application of the external remodeling simulation model can be demonstrated relative to a classical problem of cantilever-beam shape optimization. Starting from a rectangular cantilever (four elements) in transverse force loading (Fig. 3), the outside surface is remodeled in iterative steps. The remodeling objective is uniform maximal bending stress at the surface, a requirement which can be transformed to uniform SED. Figure 3 shows the results of the subsequent steps and the final shape after 15 iterations; also shown is the analytical solution, indicating good agreement.

This example indicates that these kind of predictive remodeling procedures can be considered as structural optimization methods, of which many different kinds are used in engineering mechanics. In fact, a very similar procedure was proposed by Umctani and Hirai (1975) to optimize engineering structures, which they baptised a 'growing-reforming procedure'. It is noteworthy, that these authors derived their idea for such a procedure from the theories of Roux and others, relative to adaptive behavior of bone. Interestingly, we and others are presently engaged in applying principles and methods of engineering mechanics to the problems of adaptive bone remodeling. Conversely, the above authors, in their relatively early publication, take the traditional adaptive bone remodeling theory for granted and apply it to problems of engineering mechanics. Speaking about convergence, here is an example related to the scientific process!

Characteristic of the present procedure relative to shape optimization in general is, that in the present model each material point attempts to optimize its own environment, instead of contributing to the optimization of the structure as a whole.

\section{Application to bone-density prediction}

To demonstrate the application of the internal remodeling simulation model, the classical problem of density distribution in the proximal femur is addressed. The initial configuration is a FEM model of the proximal femur with uniform density distribution, hence uniform elastic modulus $\left(E=0.5 \times 10^{4} \mathrm{MPa}\right)$,

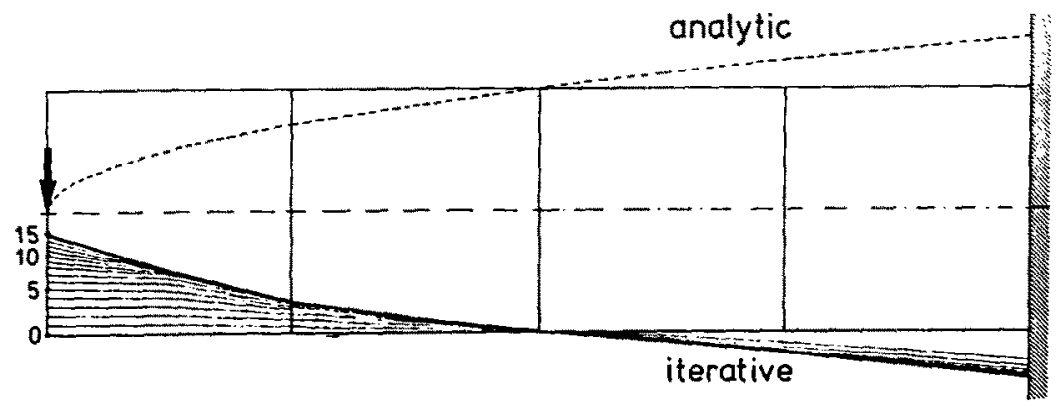

Fig. 3. Adaptive-shape optimization of a cantilever beam, loaded at the end by a transverse force, using the external remodeling model. The iterative adaptations and the final configuration predicted by the model are shown on the lower side. The analytical solution (constant SED at the surface) is shown on the upper side. 
loaded on the femoral head and by the abductor muscles. Using the internal remodeling program, the elastic modulus per element is iteratively adapted in accordance with equation (8a), based on the difference between the actual SED and a constant homeostatic SED $U_{n}$, determined as the average SED over all elements in the initial model. The elastic modulus is subject to an upper bound of $E=2.5 \times 10^{4} \mathrm{MPa}$, a high value for the modulus of cortical bone (Evans, 1973).

Figure 4 shows the resulting apparent density distribution after the 21 st iteration. For this graph, the density was calculated from the elastic modulus according to $E=a p^{2}$. where $a$ is a constant. The most extensive effects occur in the first two iterations, when the medial and lateral regions of dense bone are formed. However, notable changes in the density distribution of the inner trabecular region are also seen during the higher iterations, up to the $10-17$ th step. No notable difference is seen after the 17 th step. This information was based on graphical confirmation, no mathematical convergence criterion was used.

The result shows some similarity to the bone density distribution in the femur. The result is also very similar to the one obtained by Fyhrie and Carter (1986b) and Carter et al. (1987b), using their optimization model discussed previously, and it is interesting to compare the two theories in some more detail. The optimization function used by these authors in their 'self-

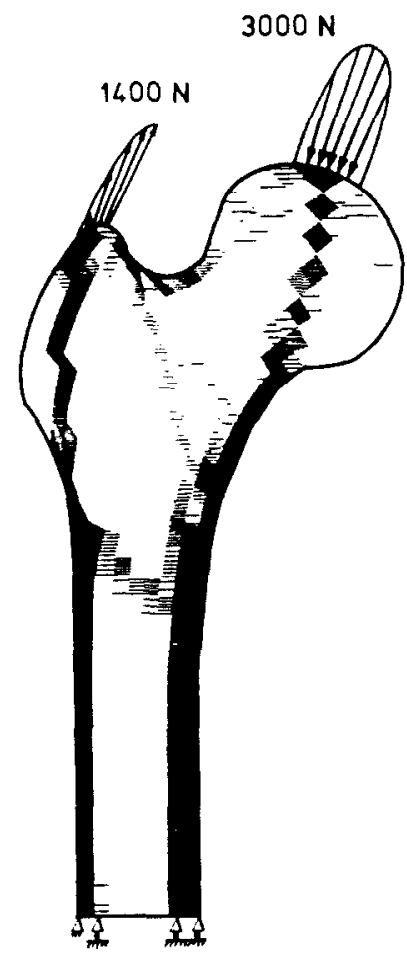

Fig. 4. Bone-density distribution in the proximal femur, for a single loading case, predicted by the internal remodeling model after 21 iterations, assuming uniform SED as the remodeling objective. optimization theory' can be written as equation (2). Obviously, in the iteration procedure with the FEM, the elastic modulus is used as the feed-back parameter, hence, considering their density/modulus relation $E=c \rho^{3}$, the optimization function transforms in

$$
E=c^{\prime \prime} U^{3} \text {. }
$$

where $c$ " is a constant. In the actual iterative process, an updated elastic modulus $E_{k}$ is determined in each element $i$ and in each step $k$, according to

$$
E_{k}^{i}=c^{\prime \prime}\left(U_{k-1}^{i}\right)^{3}, \quad 1 \leqslant i \leqslant n, \quad 1 \leqslant k \leqslant i .
$$

whereby $n$ is the number of elements, $\lambda$ the number of iterative steps, and $U_{0}^{i}$ is found with the FEM code from an initial configuration $E_{0}^{i}$.

Obviously, the theory of 'self optimization' (SO) and the present form of the theory of 'adaptive elasticity' (AE) have some distinct differences. Whereas the AEtheory is of a more conservative and general nature, describing the remodeling process of bone tissue towards a site-specific homeostatic SED $U_{n}$, the SOtheory is more specific, predicting what value $U_{n}$ should actually have. Hence, if $U_{n}=\rho / c^{\prime}$ (equation 2) would have been applied in the present example, instead of $U_{n}=$ constant, the results of both analyses should have been equal.

Both methods have in common that neither of the iterative formulas (8) and (10) guarantee convergence, although the $A E$ process, owing to its arbitrary timestep $\Delta t$ (equation 8 ), has controllable stability. In addition, the results of both methods depend on the initial configuration $E_{o}^{i}$, the geometry of the structure and the external loading characteristics. Concerning the latter aspect, Carter et al. (1987a) propose to use a weighted series of loading cases, which is probably more realistic. The sensitivity to the initial configuration at the beginning of the iterative process, i.e. the uniqueness of the solution, has not been investigated and it would be of interest to find out to which extent the two procedures are different in this respect. Finally, of course, whether the optimization criterion of the SO-theory is realistic relative to other possible optimization functions has not been shown as yet.

Similar to the situation discussed in the previous section relative to external remodeling, the $\mathrm{SO}$ and the present AE procedures both imply that each bone point optimizes its own environment, instead of contributing to the optimization of the structure as a whole. Transformed to the problem of bone architecture, this difference is equivalent to the concept of bone as a 'self-optimizing tissue' vs the concept of bone as a structure optimized by natural selection, for which the characteristics are contained in the genetic code. The vast difference in bone shapes and properties which nature has produced (Currey, 1984) pleads for the latter hypothesis. In whole-structure optimization procedures in engineering mechanics the problem can be formulated as an integral 'objective function' for the structure as a whole, which is minimized relative to an 
arbitrary amount of 'design variables' (Haug and Cea, 1981). Such a procedure gives better guarantees for convergence to a truly optimal shape of the structure as a whole.

A whole-structure optimization function derived from the theory of Fyhrie and Carter (1986a) could be formulated as

$$
F=\sum_{i=1}^{n}\left\{E^{i}-C^{\prime \prime}\left(C^{i}\right)^{3}\right\}^{2 N}, \quad 1 \leqslant i \leqslant n,
$$

where $n$ is the number of elements, to be minimized for a number of 'design variables', such as the elastic moduli (density) per element. It would be interesting to see how the result of such an optimization procedure would compare to the ones discussed above. Evidently, for a FEM procedure in a realistic case, this is an immense computer task. For an objective, wholestructure optimization, the shape of the bone should in fact be also taken into account.

\section{'STRESS SHIELDING' AND CORTICAL BONE ADAPTATION}

In clinical orthopaedics, loss of bone mass in the cortex is often seen post-operatively on X-rays after THA, in the form of cortical osteoporosis or atrophy. Although vascular and other causes for these phenomena have been suggested as well, the most popular explanation is the occurrence of 'stress shielding'. Stress shielding of bone occurs, when a load, normally carried by the bone alone, is shared with an implant. As a result, the bone stresses are subnormal and, in accordance with the theories of adaptive remodeling, the bone loses mass. Obviously, the stress-shielding vehicle in this particular case is the prosthetic stem.

The concept of stress shielding as it relates to bone resorption was originally established in combination with internal fracture fixation plates, where the plate is the stress-shielding vehicle (Tonino et al., 1976; Woo et al., 1976). Concerning the traditional cemented THA, calcar resorption at the proximal/medial femur was often thought to be related with stress-shielding (Griss et al., 1978; Oh and Harris, 1978). However, calcar resorption and other (possibly) stress-related adaptive phenomena did not seem to threaten the clinical results of cemented THA to a large extent. With the introduction of cementless prostheses, featuring boneingrowth coatings, these circumstances changed. Massive changes in bone stock were seen clinically. On the proximal side cortical atrophy would often occur, whereas an increase in cortical diameter was sometimes found near the distal tip of the stem (Lord and Bancel, 1983; Engh and Bobyn, 1985; Morscher, 1983; Brown and Ring, 1985; Engh et al., -1987). These phenomena were generally attributed to stress-related effects, based on the implications of 'Wolff's Law' in general.

Stress shielding is not really a well-understood mechanical phenomenon in clinical practice, and ad- aptive bone reactions around prostheses have not been well-defined. Usually, the bone reactions in clinical cases are reported to stabilize after about a year post operatively (Engh et al., 1987), but progressive late reactions have also been found (Brown and Ring, 1985). Sometimes the concept of 'biological fixation' by itself is believed to be responsible for bone resorption, sometimes the stiffness of the prosthesis is seen as the causative factor. In the clinical reality, these two effects are hard to separate, because porous coated stems are usually canal filling as well, hence stiff. In addition, clinical X-ray measurements of bone resorption are quite unsensitive and often inaccurate.

Animal experiments with cementless. porous coated prostheses have provided more definite information on bone resorption (Miller and Kelebay. 1981; Bobyn et al., 1982; Hedley et al., 1982; Chen et al., 1983; Dallant et al., 1986; Turner et al., 1986). In some cases, complete resorption of the cortex was found (Miller and Kelebay, 1981). Other results were less dramatic, but strongly suggested a relation with stress transfer (e.g. Bobyn et al., 1982; Hedley et al., 1982). Although the resorptive effects are often denoted as osteoporosis, several authors report only thinning of the cortex and no significant changes within the bone itself, after measurements of both (Woo et al., 1976: Dallant et al., 1986; Turner et al., 1986). However, other authors have reported cortical osteoporosis in combination with cortical atrophy after immobilization of bones in animals (e.g. Lanyon, 1984).

Investigations of the assumed relation between stress shielding and adaptive bone remodeling require assessments of both stress patterns and bone reactions. Although stress patterns in bone surrounding prostheses have been determined in a number of FEM analyses (Huiskes and Chao, 1983) and strain-gauge experiments (e.g. Oh and Harris, 1978), this information has limited value, because the analyses only represent an initial, static configuration, whereas stress-shielding and bone remodeling are transient phenomena. Using the adaptive remodeling models described in the previous section, these phenomena can be followed in time and the amount of bone resorption predicted. In the next section this approach is illustrated, whereby the effects of implant rigidity and bonding characteristics are investigated.

\section{Methods}

The remodeling procedures were applied to in vestigate the relationship between 'stress shielding' and adaptive cortical bone remodeling around an intramedullary prosthesis. To this end, a generalized, simple model of intramedullary fixation was applied (Huiskes, 1980). The FEM model (Fig. 5) is twodimensional, but simulates an axisymmetric straight bone with an axisymmetric straight stem. This is accomplished by using a side-plate element layer and by choosing its element characteristics in such a way that the dimensions in the mid-longitudinal section 


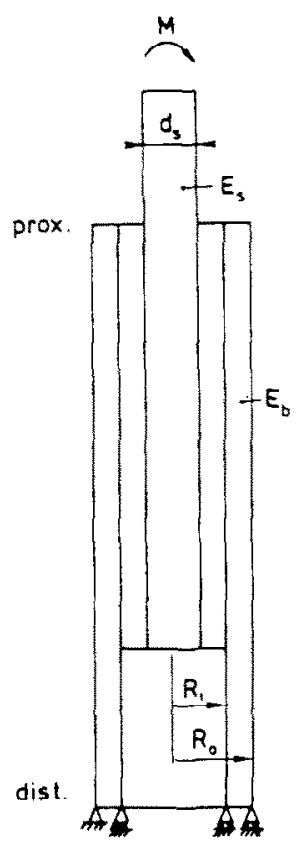

Fig. 5. The simplified, general model of intramedullary fixation applied in the remodeling analysis.

and the moments of inertia of the stem and the bone in the axisymmetric model are precisely reproduced in the two-dimensional model (see Appendix I).

In the remodeling process, it is assumed that bone adapts to the actual SED, after the implantation, by remodeling its shape or density in such a way that the pre-implantation, homeostatic SED, $U_{n}$, is obtained. In the case of internal remodeling, the difference between the actual SED and the homeostatic SED of each element is used as a feed-back signal (cf. equation 8a) to change the elastic modulus (compare Fig. 2). In the case of external remodeling, the SED in the surface nodal points is used to iteratively adapt cortical thickness (cf. equation $8 \mathrm{~b}$ ). To retain favourable element configurations, the nodal points inside the cortex are also relocated, proportional to the displacements of the surface nodal points, relative to the distance to a reference line in the structure (Fig. 6). An element is not adapted further when its width reduces to $4 \%$ of its original value. In the adaptive procedure (cf. Fig. 2), the side-plate characteristics are adapted too, in every iteration, in order to continuously retain the correspondence between the axisymmetric model and the two-dimensional FEM description, relative to the flexural rigidity (Appendix I). The underlying assumption of this procedure is, that adaptation of bone in the longitudinal section is in fact distributed evenly over the whole cross-section in an axisymmetric description.

To determine the external load, the 'loading history' theory of Carter et al. (1987a) is adopted. It is assumed that in the pre-implantation stage, the bone cortex is in an homeostatic condition, which implies that, relative

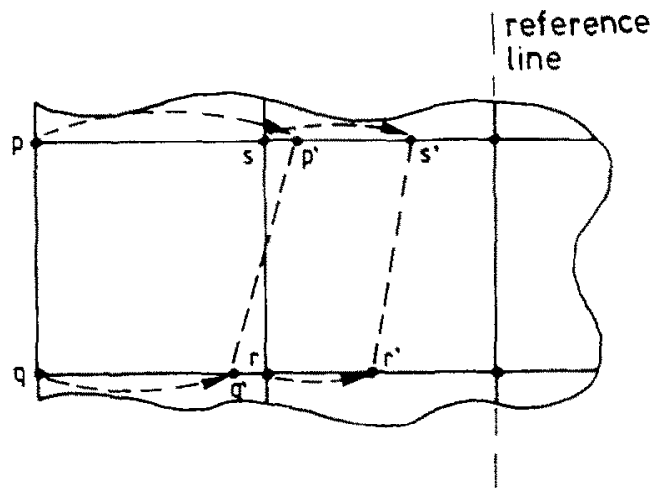

Fig. 6. Relocation of external and internal nodal points, relative to a reference line of the structure, in order to maintain adequate element shapes.

to a representative 'daily' loading cycle $1 \leqslant j \leqslant c$ the stimulus for bone activity (Carter et al., 1987a)

$$
\left(\sum_{j=1}^{c} n_{j} \sigma_{e f \delta j}^{M}\right)^{1 / 2 M}=\text { constant }
$$

If we assume $M=2$ (Whalen et al, 1986), this transforms to

$$
\left(\sum_{i=1}^{c} n_{j} U_{j}\right)^{1 / 4}=\text { constant }
$$

since $\sigma_{* f f}^{2}=2 E U$ (Carter et al., 1987). Taking the three representative femoral loading cases of a daily cycle suggested by Carter et al. (1987a, b), and $n_{1}=n_{2}=n_{3}$ $=1$, it turns out (Appendix II) that the condition (12b) in the cortex can be approximately realized by a pure bending moment $M$, which can be considered as a 'substitute load' for an homeostatic loading cycle.

This choice can also be justified from another angle. As shown by Huiskes et al. (1981), the predominant loading mode of the femoral diaphysis is due to bending. In fact, the axial force component in the diaphysis contributes very little to the SED in the cortex. Since the bone is assumed as a straight tube in this case, and this form presumably represents an homeostatic condition (uniform SED), the corresponding load would be pure bending.

In the calculations, internal and external remodeling were simulated separately, whereby the stem diameter, the remodeling threshold level and the implant/bone contact conditions were varied (see Table 1). In the case of the small and the intermediate stem diameters, an acrylic cement layer was assumed between the stem and the bone. The value for the homeostatic SED, $U_{n}$, in the surface nodal points is shown in Table 1. Inside the elements (average per element) its value is somewhat lower. In the case that the implant is assumed loose from the bone, gap elements are applied, whereby no shear or tension can be transmitted at the interface, but only compression. After each bone remodeling iteration, the nonlinear contact conditions are solved iteratively, using a Newton-Raphson procedure (Klever, 1984). 
Table 1. Values and variations of parameters in the model of Fig. 5

\begin{tabular}{llll}
\hline Parameter & Symbol & Unit & Values (s) \\
\hline Bending moment & $M$ & $\mathrm{Nmm}$ & 1000 \\
Outer bone diameter & $2 r_{a}$ & $\mathrm{~mm}$ & 30 \\
Inner bone diameter & $2 r_{i}$ & $\mathrm{~mm}$ & 20 \\
Stem length in bone & $L$ & $\mathrm{~mm}$ & 80 \\
Stem diameter* & $d_{s}$ & $\mathrm{~mm}$ & Variable: $10,13.3$ and 20 \\
Bone modulus & $E_{b}$ & $\mathrm{MPa}$ & $2 \times 10^{4}$ \\
Cement modulus & $E_{t}$ & $\mathrm{MPa}$ & $2 \times 10^{3}$ (if present) \\
Stem modulus & $E_{s}$ & $\mathrm{MPa}$ & $2 \times 10^{3}$ \\
Poissons' ratios & $y$ & - & 0.3 \\
Stem flex. rigidity* & $F_{s}=E_{s} l_{s}$ & $\mathrm{Nmm}$ & Variable: $0.98 \times 10^{8}, 3.07 \times 10^{8}, 15.7 \times 10^{8}$ \\
Remodeling threshold & $s$ & $\%$ & Variable: $0,5,15,30$ \\
Natural SED & $U_{n}$ & $\mathrm{MPa}$ & S.03 $\times 10^{-6}$ (at periosteal bone surface) \\
Implant/bone interface & - & & Variable: (1) rigidly connected (2) loose (no \\
& & & tension, no friction) \\
\hline
\end{tabular}

*A stem of $d_{s}=10.8 \mathrm{~mm}, F_{3}=1.32 \times 10^{8} \mathrm{Nmm}^{2}$ was used in one case of internal remodeling.

\section{Results}

The adaptive (external) remodeling process for the fixed intermediate stem $\left(d_{s}=13.3 \mathrm{~mm}\right)$ and no remodeling threshold $(s=0)$ is illustrated in Fig. 7. As a consequence of the geometry and the bending load, both cortices behave symmetrically. After implantation of the stem, the SED at the surface of the bone is reduced relative to the natural value $U_{n}$, in particular at the proximal side (curve 0 in Fig. 7a). This reduction represents the stress-shielding effect. Bone is then removed from the outer surface in the first remodeling step (curve 1 in Fig. 7b), which causes an increase in the SED (curve 1 in Fig. 7a). This process continues until, after the ninth step, the bone surface has virtually

(a)
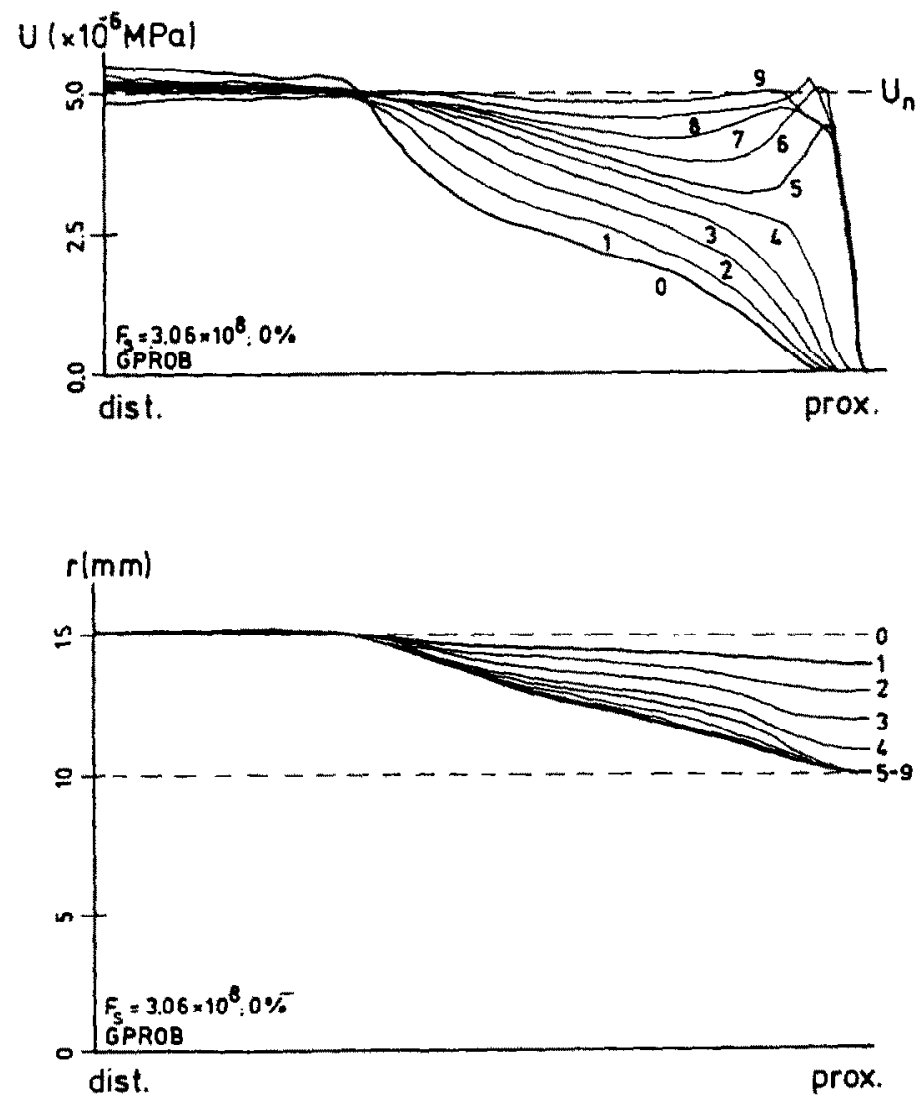

(b)

Fig. 7. Development of bone SED (a) and reduction of bone outer radius (b) during external remodeling (intermediate stem diameter, bonded interface, zero threshold level). 
regained the homeostatic SED value $U_{n}$. Hence, the bone reaches a homeostatic configuration after a wedge-formed volume of about half the cortex has resorbed (Fig. 7 b).

In the case of the thick, canal filling cementless stem, shown in Fig. $8\left(d_{s}=20 \mathrm{~mm}, s=5^{\circ}\right)$, the degree of stress-shielding is much higher (Fig. 8a). Subsequent bone resorption (Fig. 8b), however, does not result in a significant increase of the SED (Fig. 8a). In this case the bone resorption process continues until, after five iterations, virtually all bone adjacent to the stem has disappeared, and the process is terminated.

The final configurations for the three stem thicknesses are illustrated in Fig. 9. The most flexible stem causes some bone resorption at the proximal end only, somewhat similar to what is called 'calcar resorption' in clinical orthopaedics. Evidently, the differences in bone resorptive reactions are results of the differences in stem rigidity only.

The effects of the threshold level $s$ are quite predictable: when $s$ is large, the resorption process develops more slowly and causes bone resorption to a somewhat lesser extent in the flexible-stem cases. Threshold levels up to $s=30 \%$ cannot save the bone in the case of the cementless stem, as could already be predicted from the degree of stress shielding in Fig. 8.

When the stem is assumed loose, the resorption process develops somewhat differently (Figs 10 and
11). In this case the medial and lateral cortices are not stress shielded to the same degree, hence the resorption process is asymmetric. As a result, the lateral cortex, which is more severely stress shielded, resorbs faster than the medial one, which, after five iterations, starts serving as a console to sustain the proximal part of the stem. Due to high stresses on the lateral/distal side, near the stem tip. the lateral cortex increases in thickness. For the most flexible stem $\left(d_{s}=10 \mathrm{~mm}\right.$, $s=5 \%$ this effect is illustrated in Fig. 10. As a reaction to the most rigid stem, all proximal bone resorbs, as in the case of the fixed stem. Extensive cortical thickening occurs at the distal side (Fig. $11 ; d_{s}=20 \mathrm{~mm}, s=5 \%$ ) Homeostatic conditions are reached in neither of these two examples, as evident from the SED curves in Figs 10 and 11. It is uncertain as yet whether the continuation of the process would result in a stable end configuration.

In the case that internal remodeling by adaptation of the elastic modulus was simulated, the results were quite different. This process turned out to be much less effective for the bone. A homeostatic end configuration was reached only in the case of the fixed implant, the most flexible stem $\left(d_{s}=10 \mathrm{~mm}\right)$, and assuming the maximal threshold level $s=30 \%$, in which case only the proximal $1 / 3$ of the bone was fully demineralized. An illustration of the internal remodeling process is shown in Fig. $12\left(d_{s}=10.8 \mathrm{~mm}, F_{s}=1.32\right.$

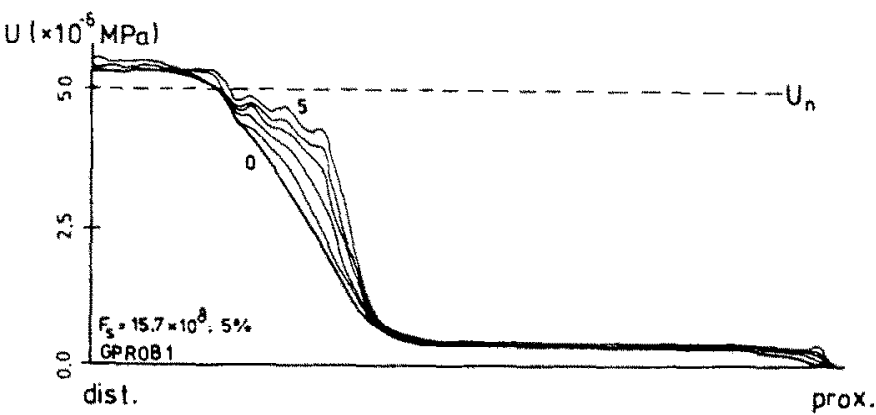

(b)

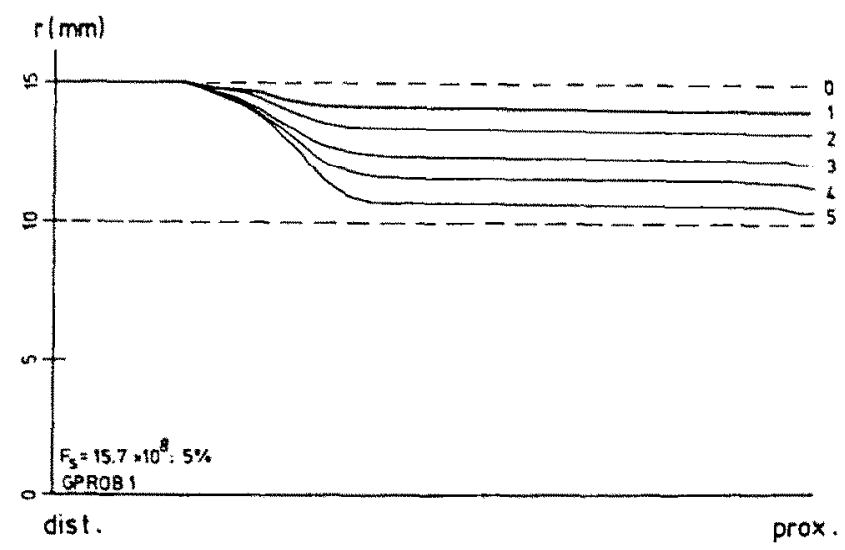

Fig. 8. Development of bone SED (a) and reduction of bone outer radius (b) during external remodeling (canal-filling stem, bonded interface, $5 \%$ threshold level). 

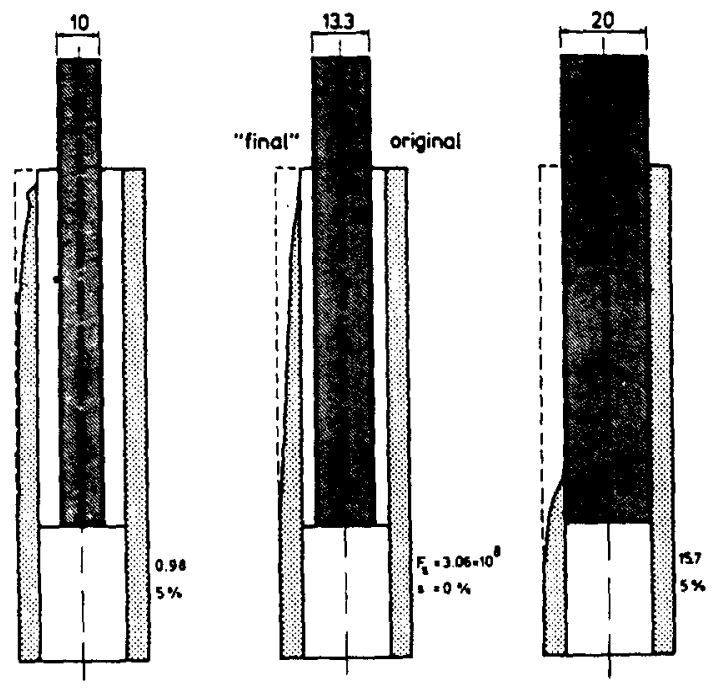

Fig. 9. Final configurations of the cortices after external remodeling around three stems of increasing diameter. In the case of the canal-filling stem, the iterative process was terminated after five steps (compare Fig. 8). $\times 10^{8} \mathrm{Nmm}^{2}, s=0 \%$ ). Evidently, massive bone resorption hardly increases the SED in this case. The iteration was terminated after six steps. when it became clear that SED normalisation would not be obtained. Even more dramatic effects occur when the canalfilling cementless prosthesis is applied $\left(d_{s}=20 \mathrm{~mm}\right)$. In this event of extreme stress shielding the SED even decreased for progressive bone resorption. In retrospect, it may have been more realistic to utilize the 'true' or bulk SED $U_{b}=\rho_{c} U / \rho$ as the feed-back variable in these cases of internal remodeling (Fyhrie and Carter, 1986b; Carter et al., 1987a).

\section{Analytical considerations}

The results presented in the previous section can be understood better with the aid of a simple analytical model. As shown earlier (Huiskes, 1980), the intramedullary fixation model used here behaves approximately in accordance with composite beam theory in the middle-stem region, where a plateau occurs in the SED-curves (Figs 7, 8, and 12). Assuming an axisymmetric configuration, the maximal SED at the surface in this region can be expressed (Huiskes, 1980) as

$$
U=\left(1-v^{2}\right) \frac{E r_{0}^{2}}{2\left(E I+F_{s}\right)^{2}} M^{2}
$$
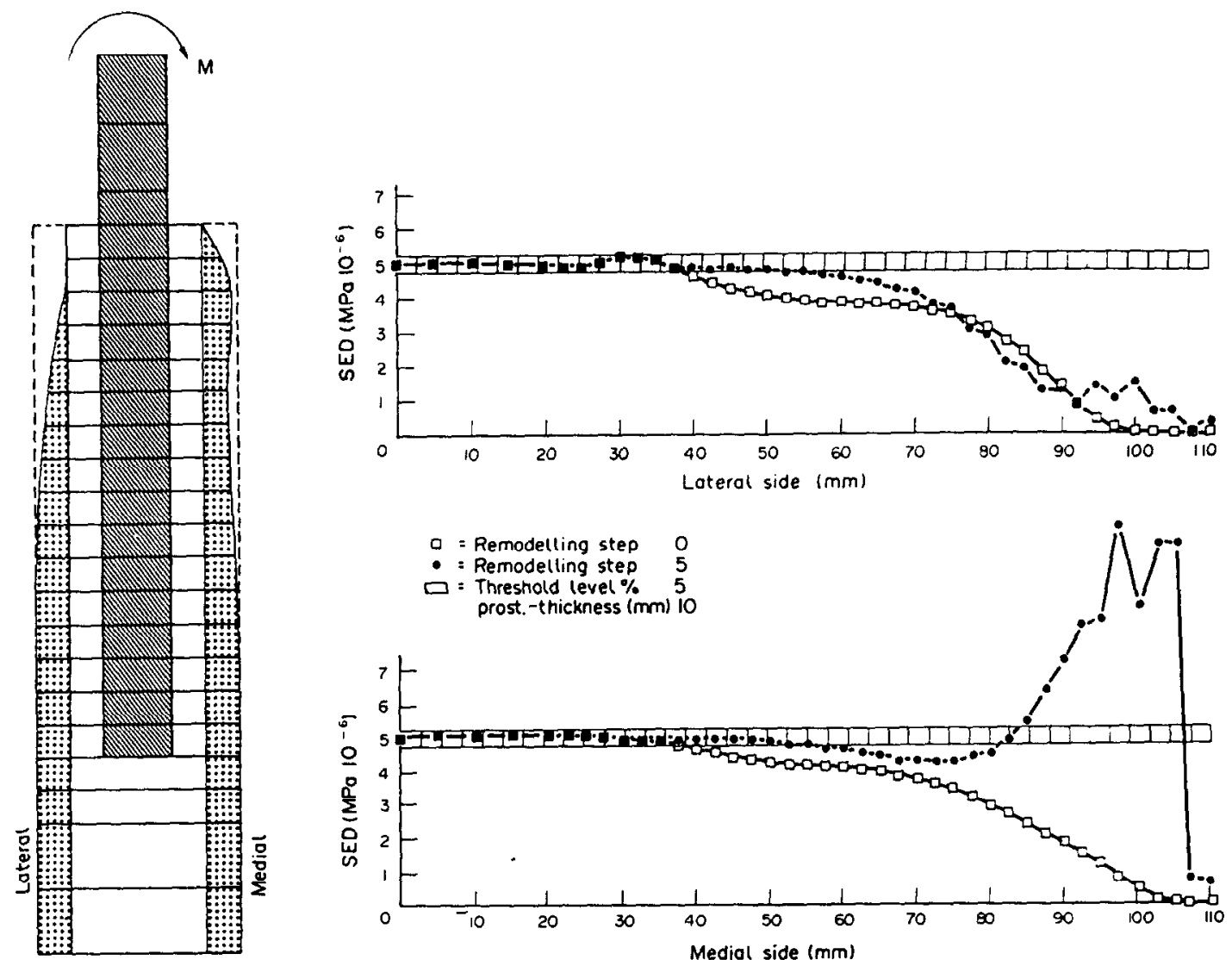

Fig. 10. Cortical configuration at the medial and proximal sides around the most flexible, loose stem, predicted by external remodeling after five iterations. The initial and final SED distributions are shown as well. 

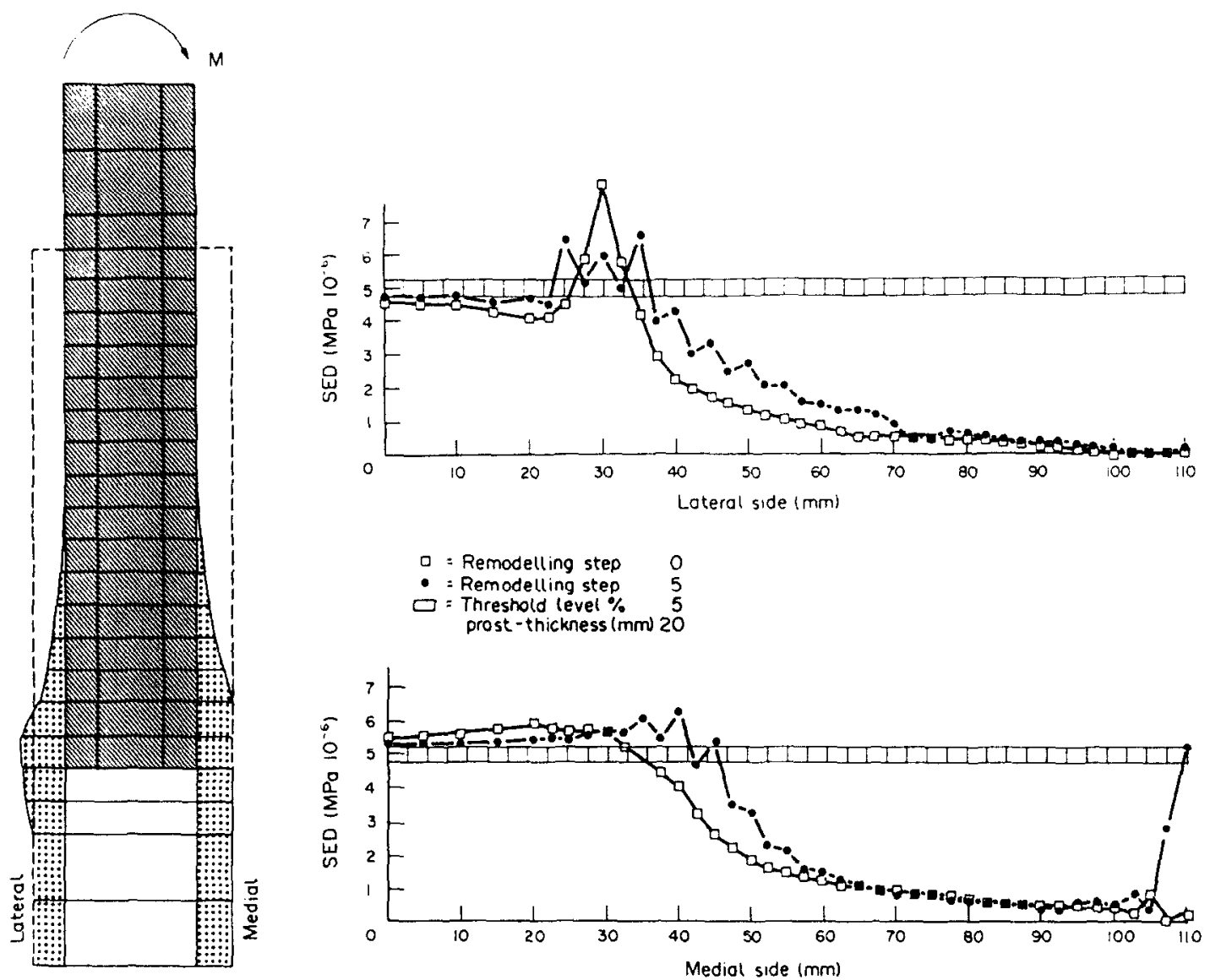

Fig. 11. Cortical configuration at the medial and proximal sides around the most rigid, loose stem, predicted by external remodeling after five iterations. The initial and final SED distributions are shown as well.

where $E=E_{b}$ and $I=\left(r_{0}^{+}-r_{i}^{+}\right) \pi / 4$ is the moment of inertia of the bone. The flexural rigidity of the stem $F_{s}=E_{s} I_{s}$ represents in fact the stress-shielding factor in this formula. Evidently, $U$ decreases monotonically with increasing $F_{s}$, and for $F_{s}=0$ the SED $U=U_{n}$, the homeostatic SED.

External remodeling implies an adaptation of $r_{0}$, hence also of $I$ in equation (13). Internal remodeling implies an adaptation of the elastic modulus $E$. It is evident from this formula that the effectiveness of these bone adaptations towards SED normalization depends on the value of the stem rigidity $F_{s}$; if $F_{s}$ overshadows $E I$, the SED becomes proportional to $E r_{i}^{2}$, hence decreases for decreasing $E$ and decreasing $r_{0}$. These effects are shown graphically in Fig. 13, presenting the SED $U$ as a function of the outer bone radius $r_{o}$ for constant $E$, and several values of $F_{s}$ (Fig. 13a), and $U$ as function of $E$ for constant $r_{o}$, and several values of $F_{s}$ (Fig. 13b). In both cases, parameter values as in Table I were taken. SED normalization is feasible, according to these curves, up to certain values of $F_{s}$. Evidently, this boundary value is much higher for external remodeling (Fig. 13a) than it is for internal remodeling (Fig. 13b). Hence, the external remodeling process is more effective, and internal remodeling will lead to progressive bone resorption for a much lower degree of stress shielding, which was also evident in the simulation analysis.

From equation (13) the boundary values for the stem rigidity $F_{s}$ in general can be determined for both remodeling modes. It is found that, according to this simple model, SED normalization (homeostasis) by external remodeling cannot be obtained when

$$
F_{s}>\frac{r_{i}}{r_{0}} E I(1-s)^{-1 / 2},
$$

and SED normalization by internal remodeling cannot be obtained when

$$
F_{s}>\frac{1}{4} E I(1-s)^{-1}
$$

Evidently, these predictions are of a rough, approximate nature, because of the simplicity of the model. However, they are compatible with the results of the simulation analyses in an approximate sense, and at least give some sort of first-order guideline.

\section{DISCUSSION}

It must be appreciated that the FEM model utilized here is a simple, general one relative to the actual 


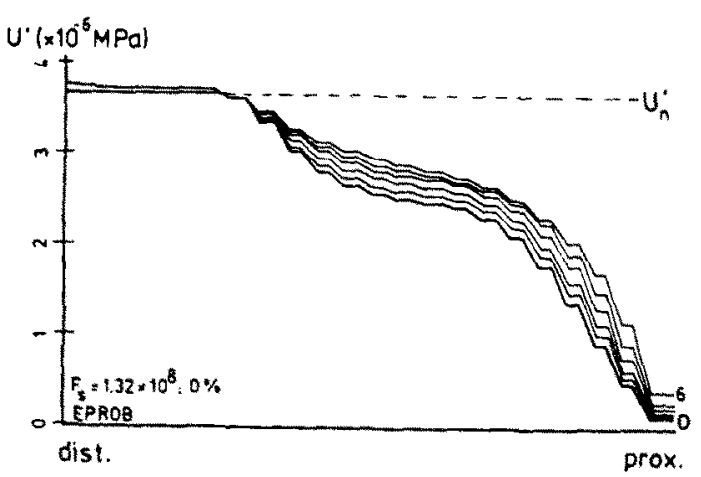

(a)

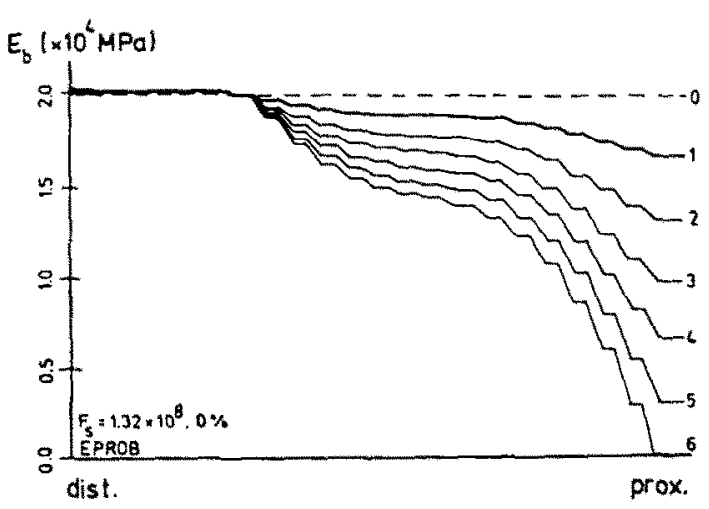

(b)

Fig. 12. Development of the SED (a) and the reduction of the elastic bone modulus (b) during internal remodeling (intermediate stem diameter $\left(d_{s}=10.8 \mathrm{~mm}\right)$, bonded interface, zero threshold level).

THA configuration. However, it serves well to illustrate the potential applicability of the adaptiveremodeling simulation models to prosthetic-design analysis, and at least some general tendencies of the stress-shielding phenomenon can be clarified. Although simple, it should be noted that the representation of the femoral diaphysis by a cylinder to evaluate approximate stress patterns is not unreasonable (Huiskes et al., 1981). The side-plate FEM representation is adequate to describe the threedimensional structural integrity of the bone in this case (Huiskes, 1980), and external loading by a pure bending moment is a reasonable, general representation of the most significant effects of hip-joint and abductor muscle loading in the diaphysis (Huiskes et al., 1981; Rohlmann et al., 1983; Appendix II). The model is less realistic for the loose-stem condition, because in that case the actual load-transfer mechanism depends greatly on the axial hip-joint force component and the actual geometry of the interface (Weinans et al., 1987).

Several assumptions are applied in the present approach to bone-remodeling analysis. The choice for SED as the feed-back control variable for adaptive remodeling is in fact an arbitrary one, but well- defendable on physical grounds (Carter et al., 1987a). It is a physical quantity for which it is possible to conceive mechanisms for its measurement by bone. This is not true of stress. It has also a relation to both rigidity and strength. From a methodological standpoint it has the advantage of being a scalar variable and an invariant of the strain tensor, which implies that only one remodeling coefficient $\left(C_{x}\right.$ or $\left.C_{x}\right)$ must be established, as opposed to the many coefficients needed by Cowin and associates in the original theory of adaptive elasticity. The theory, as it is presently used, is a static one, only considering the absolute SED values, not their rate of change. Although it is unlikely that static load-changes result in adaptive bone reactions (Rubin and Lanyon, 1984), the philosophy is, to consider the SED amplitudes as general representations of the actual recent loading history. This philosophy was also adopted by Carter et al. (1987a), who proposed to use series of loading magnitudes. As shown in the present analysis, a substitute single loading case can be found to represent the SED effects of such a series, at least where it concerns the cortical bone.

It was assumed in the adaptive remodeling analysis that all changes in SED levels between pre- and postimplantation were caused by the implant itself, and not by changes in the external loads. Although this seems to be a reasonable assumption, in view of the fact that the load reflects the integrated effects of a series of typical daily activities, which is usually also implicitly accepted in clinical reports about effects of "stress shielding, it is not obvious that this is actually true. Evidently, the majority of patients have a more active motion pattern in the postoperative as compared to the preoperative period: pain and functional limitations are precisely the reasons for which they receive a THA. This is different in animal experiments, which are commonly performed on healthy (and physiologically younger) animals. This may be one of the reasons why adaptive phenomena around implants are more prominent in animal experiments (Miller and Kelebay, 1981; Bobyn et al., 1982; Hedley et al., 1982; Chen et al., 1983; Dallant et al., 1986; Turner et al., 1986) than they are in patient material (Lord and Bancel, 1983; Engh and Bobyn, 1985; Morscher, 1983; Brown and Ring, 1985; Engh et al., 1987). For this reason, the present results are probably more directly related to these animal experiments than they are to clinical series.

The most important fruits from the present analysis are the falsifiable, quantitative hypotheses relative to the predicted effects of stem rigidity and degree of fixation. The present results are compatible with the animal-experimental data on similar intramedullary configurations reported by Miller and Kelebay (1981), Chen et al. (1983), Dallant et al. (1986) and Turner et al. (1986). In particular the finding of a completely resorbed cortex around canal-filling. well-fixed prostheses by Miller and Kelebay (1981), compares well with the present predictions for such a configuration. The frequent finding of calcar resorption in the case of 
(a)

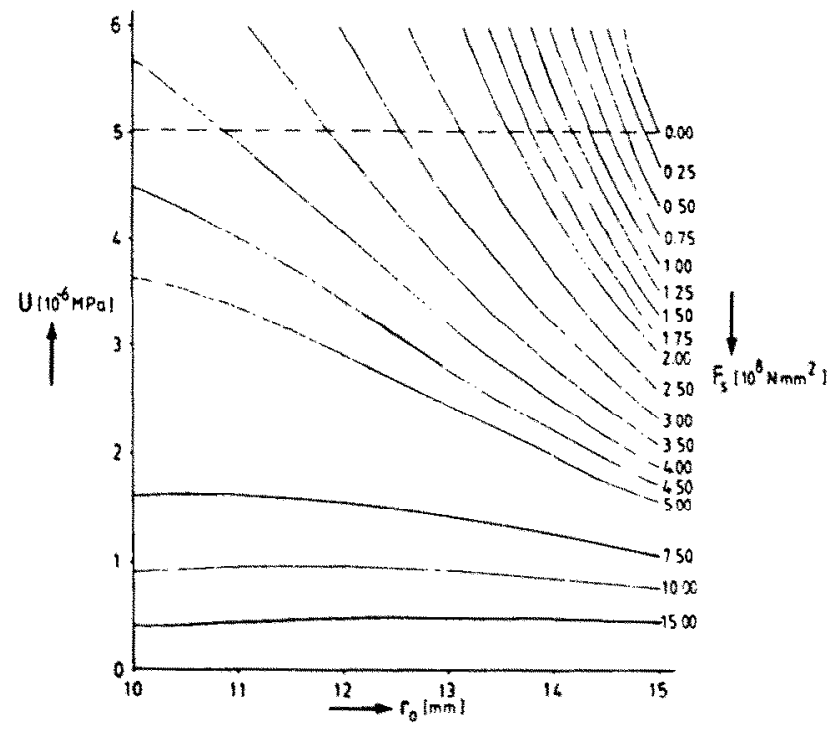

(b)

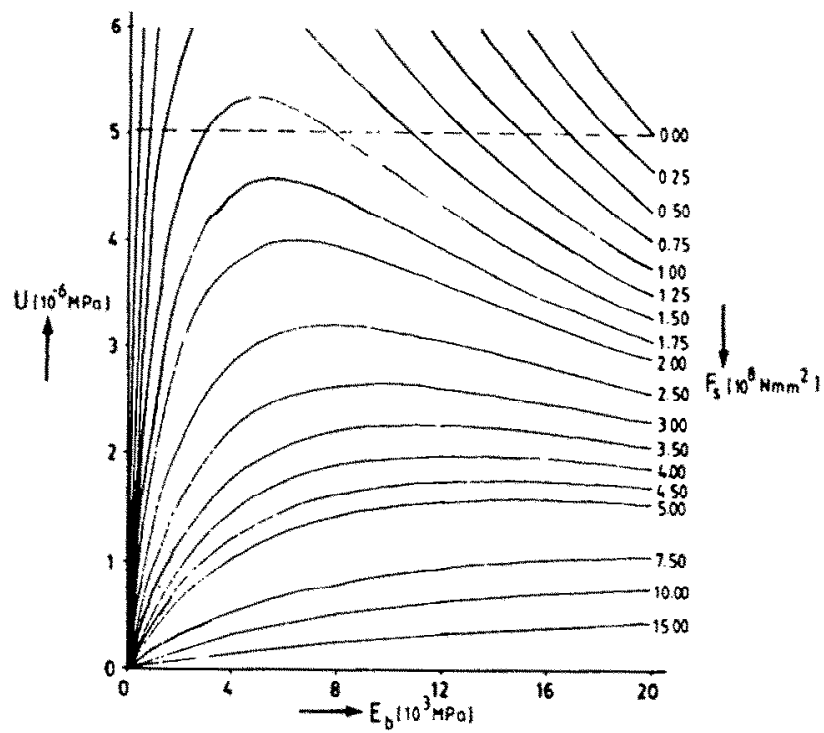

Fig. 13. SED at the bone surface, for variable stem rigidities, (a) as a function of the outer bone radius, and (b) as a function of the elastic modulus (composite-beam model). The homeostatic SED of $5.03 \times 10^{-6} \mathrm{MPa}$ is indicated.

less rigid (cemented) prostheses is also predicted with the present method.

The relationship between bone loading and adaptive cortical remodeling in general has been well established in animal experiments (e.g. Rubin and Lanyon, 1984). There is no reason to assume that when bone strains are reduced by implants (stress shielding), rather than by a reduction of external loads, bone would behave differently; indeed, many experiments with fracture fixation plates and intramedullary prostheses, cited above, have indicated that, in this case too, a relationship is evident. The present analyses, based on a quantiative description of this relationship, show that 'stress shielding" is a transient phenomenon, and that a homeostatic strain configuration can in principle be obtained by the bone, provided that the stem is not too rigid, and provided that the bone resorption process takes place mainly at the periosteal surface, rather than inside the cortex. To decide whether a stem is too rigid to make bone-strain normalisation likely, approximate quantitative guidelines were derived (equations 14 and 15). Although based on a very simple model, these guidelines are quite compatible with the clinical results on cementless porous coated prostheses reported by Engh and Bobyn (1985).

The present results also predict that the adaptive 
remodeling process will be quite different in the case of loose prostheses as compared to well-fixed (ingrown) prostheses. A finding which is in agreement with the animal-experimental results of Turner et al. (1987). To what extent, precisely, the process will be different in a realistic situation cannot readily be predicted from the present analysis because the straight stem model is not a very good one to describe in particular the proximal part of the a loose-stem THA configuration (Weinans et al., 1987). In any case, it appears from the analysis that an increase of cortical thickness near the tip of the stem, as often reported in the literature, is associated with relatively rigid stems, which are not well-bonded to the bone.

It is obvious that much work is still to be done on the development of the quantitative bone-remodeling theories, their experimental verification, and the numerical simulation methods actually applied. Important issues, in this respect, are the convergence of the numerical procedures and the uniqueness of the solutions, under the conditions assumed. As discussed earlier, many important theoretical concepts and controversies have not yet been resolved, as for instance the theory of self-optimization versus genetic predestination (and their inter-relationship!). It is evident, however, that the gap between mechanical analysis and living bone is rapidly closing.

It is interesting to note that more than 100 years passed between the creation of 'Wolff's Law' and its quantitative application for practical purposes. In retrospect, it is evident that a necessary step for this to happen was the characterization of bone as a mechanical structure and as material. On both accounts, pioneering research was conducted by F. Gaynor Evans (1957, 1973).

Acknowledgement-Sponsored in part by the Netherlands Organisation for the Advancement of Pure Research (ZWO/MEDIGON). We are grateful to Dennis R. Carter, Ph.D., and his associates, for providing some details of their analyses not contained in their articles, and by drawing our attention to the publication of Umetani and Hirai (1975).

\section{REFERENCES}

Bobyn, J. D., Cameron, H. U., Abdulla, D., Pilliar, R. M. and Weatherly, G. C. (1982) Biologic fixation and bone remodeling with an unconstrained total knee prosthesis. Clin. Orthop. Rel. Res. 166, 301-312.

Brown, I. W. and Ring, P. A. (1985) Osteolytic changes in the upper femoral shafi following porous coated hip replacement. J. Bone Jt Surg. 67-B, 218-221.

Carter, D. R. (1984) Mechanical loading histories and cortical bone remodeling. Calcif. Tissue Int. 36, S19-S24.

Carter, D. R., Fyhrie, D. P. and Whalen, R. T. (1987a) Trabecular bone density and loading history: regulation of connective tissue biology by mechanical energy. J. Biomechanics 20, 785-794.

Carter, D. R. and Hayes, W. C. (1977) The behavior of bone as a two-phase porous structure. J. Bone Jt Surg. 59-A, 954-962.

Carter, D. R., Orr, T. E., Fyhrie, D. P., Whalen, R. T. and Schurman, D. J. (1987b) Mechanical stress and skeletal morphogenesis, maintenance, and degeneration.
Transactions 33rd Annual Orthopedic Research Societ p. 462.

Chen, P. Q., Turner, T. M., Ronningen, H., Galante, J., Urban. R. and Rostoker, W. (1983) A canine cementless total hip prosthesis model. Clin. Orthop. Rel. Res. 176, 24-33.

Cowin, S. C. (1986) Wolfr's law of trabecular architecture at remodeling equilibrium. J. biomech. Engng 108, 83-88.

Cowin, S. C. and Firoozbakhsh, K. (1981) Bone remodeling of diaphyseal surfaces under constant load: theoretical predictions. J. Biomechanics 14, 471-484.

Cowin, S. C., Hart, R. T., Baber, J. R. and Kohn, D. H. (1985) Functional adaptation in long bones: establishing in vito values for surface remodeling rate coefficients. J. Biomechanics 18,665-684.

Cowin, S. C. and Hegedus, D. H. (1976) Bone remodeling I: theory of adaptive elasticity. J. Elasticity 6, 313-326.

Currey, J. (1984) The Mechanical Adaptations of Bone. Princeton University Press, Guildford

Dallant, P., Meunier, A., Guillemin, G., Christel, P., Crolet, J. M. and Sedel, L. (1986) Cortical bone response to orthopaedic implant rigidity: an experimental study. Biological and Biomechanical Performance of Biomaterials (Edited by Christel, P., Meunier, A. and Lee, A. J. C.). pp. 441-446. Elsevier Science, Amsterdam.

Engh, C. A. and Bobyn, J. D. (1985) Biological Fixation in Total Hip Arthroplasty. Slack. New Jersey.

Engh, C. A., Bobyn, J. D. and Glassman, A. H. (1987) Porous coated hip replacement: the factors governing bone ingrowth, stress shielding, and clinical results. J. Bone $J t$ Surg. 69-B, 45-55.

Evans, F. G. (1957) Stress and Strain in Bones. Charles C. Thomas, Springfield. IL.

Evans, F. G. (1973) Mechanical Properties of Bone. Charles C. Thomas, Springfield, IL.

Firoozbakhsh, K. and Cowin, S. C. (1981) An analytical model for Pauwels' functional adaptation mechanism in bone. J. biomech. Engng 103, 247-252.

Frost, H. M. (1964) The Laws of Bone Structure. Charles C. Thomas, Springfield, IL.

Fyhrie, D. P. and Carter, D. R. (1986a) A unifying principle relating stress to trabecular bone morphology. J. Orthop. Res. 4, 304-317.

Fyhrie, D. P. and Carter, D. R. (1986b) Prediction of cancellous bone apparent density with 3-D stress analysis. Transactions 32nd Annual Orthopedic Research Society, p. 331.

Griss, P., Heimke, G., Werner, E., Bleicher, J. and Jentschwa, G. (1978) Was bedeutet die Resorption des Calcar Femoris nach der Totalendoprothesenoperation der Huefte? Archs Orthop. Traumat. Surg. 92, 225-232.

Hart, R. T., Davy, D. T. and Heiple, K. G. (1984) A computational method of stress analysis of adaptive elastic materials with a view toward application in strain induced remodeling. J. biomech. Engng 106, 342-350.

Haug, E. J. and Cea, J. (Eds) (1981) Optimization of Distributed Parameter Structures. Sijthoff and Noordhoff, Alphen a/d Rijn.

Hayes, W. C. and Snyder, B. (1981) Toward a quantitative formulation of Wolf's law in trabecular bone. Mechanical Properties of Bone (Edited by Cowin, S. C.), AMD-Vol. 45, pp. 43-69. The American Society of Mechanical Engineers, New York.

Hedley, A. K., Clarke, I. C., Kozinn, S. C., Coster, I., Gruen, I. and Amstutz, H. C. (1982) Porous ingrowth fixation of the femoral component in a canine surface replacement of the hip. Clin. Orthop. Rel. Res. 163, 300-311.

Hegedus, D. H. and Cowin, S. C. (1976) Bone remodeling II: small strain adaptive elasticity. J. Elasticity $6,337-352$.

Huiskes, R. (1980) Some fundamental aspects of human-joint replacement. Acta Orthop. Scand. Suppl. 185.

Huiskes, R. and Chao, E. Y. S. (1983) A survey of finite element methods in orthopaedic biomechanics. J. Biomechanics 16, 385-409. 
Huiskes. R.. Janssen. J. D. and Slooff T. J. (1981) A detailed comparison of experimental and theoretical stress-analyses of a human fernur. Mechanical Properties of Bone (Edited by Cowin. S. C.). AMD-Vol 45. pp. 211-234. American Society of Mechanical Engineers, New York.

Klever, F. J. (1984) On the mechanics of failure of artificial knee joints. Ph. D. Dissertation, University of Twente. The Netherlands.

Koch. J. C. (1917) Laws of bone architecture. Am. J. Anat. 21, 177-298.

Kummer. B. (1972) Biomechanics of bone: mechanical properties. functional structure and functional adaptation. Biomechanics, its Foundations and Objectives (Edited by Fung. Y. C., Perrone, N. and Anliker, M.) pp. 237-272. Prentice Hall, Englewood Clifs, NJ.

Lanyon. L. E. (1984) Functional strain as a determinant for bone remodelling. Calcif. Tissue Int. 36, S56-S61.

Lord, G. A. and Bancel, P. (1983) The madreporic cementless total hip arthroplasty: new experimental data and seven year clinical follow-up study. Clin. Orthop. Rel. Res. 176, $67-76$.

Miller, J. E. and Kelebay, L. C. (1981) Bone ingrowth-disuse osteoporosis. Orthop. Trans. 5, 380.

Morscher. E. (Ed.) (1983) Die zementlose Fixation von Hueftendoprothesen. Springer. Berlin.

Oh, I, and Harris. W. H. (1978) Proximal strain distribution in the loaded femur. J. Bone Jt Surg. 60-A, 75-85.

Pauwels. F. (1965) Gesammelte Abhandlungen zur funktionellen Anatomie des Bewegungsapparates. Springer, Berlin.

Roesler. H. (1981) Some historical remarks on the theory of cancellous bone structure (Wolrt's law). Mechanical Properties of Bone (Edited by Cowin, S. C.), AMD-Vol. 45, pp. 27+2. American Society of Mechanical Engineers, New York.

Rohlmann. A.. Moessner, U.. Bergmann, G. and Koelbel, R. (1983) Finite element analysis and experimental investigation in a femur with hip endoprothesis. J. Biomechanics 16 , $727-742$.

Rubin, C. T. and Lanyon, L. E. (1984) Regulation of bone formation by applied dynamic loads. J. Bone Jt Surg. 66-A, $397-402$.

Tonino, A. J., Davidson, C. L.. Klopper, P. J. and Linclau, L. A. (1976) Protection from stress in bone and its effects. $J$. Bone Ji Surg. 58-B, 107-133.

Turner, T. M., Sumner, D. R., Urban, R. M. and Galante, J. O. (1987). Femoral remodeling associated with uncoated and porous coated cementless total hip arthroplasty. Proceedings 33rd Annual Meeting Orthopedic Research Society: p. 408.

Turner, T. M., Sumner, D. R., Urban, R. M., Rivero, D. P. and Galante. J. O. (1986) A comparative study of porous coatings in a weight-bearing total hip arthroplasty model. J. Bone Jt Surg. 68-A, 1396-1409.

Umetani, Y. and Hirai, S. (1975) An adaptive shape optimization method for structural material using the growingreforming procedure. Proceedings 1975 Joimt JSMEASME Applied Mechanics Western Conference, pp. $359-365$.

Weinans, H., Huiskes, R. and Grootenboer. H. (1987) The modeling and mechanical. consequences of fibrous-tissue formation around femoral hip prostheses. 1987 Biomechanics Symposium (Edited by Butler, D. L. and Torzilli. P. A.) AMD-Vol. 84, pp. 89-92. American Society of Mechanical Engineers, New York.

Whalen, R. T, Carter, D. R. and Steele, C. R. (1986) The relationship between physical activity and bone density. Transactions 33rd Annual Meeting Orthopedic Research Society. p. 463.

Wolff. J. (1869) Ueber die Bedeutung der Architeklur der spongiösen Substanz. Zent bl. med. Wiss. VI, 223-234.

Woo, S. L.-Y., Akeson, W. H., Coutts, R. D., Rutherford, L., Doty. D. Jemmott. G. F. and Amiel, D. (1976) A comparison of cortical bone artrophy secondary to fixation with plates with large differences in bending stiffness. $J$. Bone Ji Surg. 58-A, 190-195.

\section{APPENDIX 1. CHARACTERISTICS OF THE SIDE-PLATE MODEL}

The element thicknesses of the side-plate model are selected and adapted in such a way, that the correspondence with an axisymmetric bone model is continuously maintained, with respect to the geometry in the longitudinal section $\left(r_{b}, r_{0}\right)$ and the moment of inertia (l). The relation used for this purpose is shown in Fig. Al: when the outer cortical diameter changes, the thickness of the cortical elements, $r_{0}$, and the thickness of the side-plate elements, $r_{\mathrm{o}}-d$, are adapted accordingly. A similar procedure is applied for internal remodeling with respect to the elastic modulus of the cortical and side-plate elements.
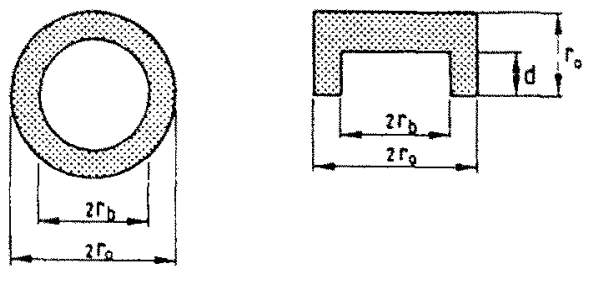

$$
\begin{aligned}
& I_{0}=\frac{\pi}{4}\left(r_{0}^{6}-r_{0}^{6}\right) \quad I_{0}=\frac{1}{12}\left(r_{0}\left(2 r_{0}\right)^{3}-d\left(2 r_{b}\right)^{3}\right) \\
& I_{0}=I_{0} \Rightarrow d=\frac{1}{8 r_{0}^{3}}\left(3 \pi r_{b}^{6}-(3 \pi-8) r_{0}^{4}\right)
\end{aligned}
$$

Fig. A1. Relation between moments of inertia in the axisymmetric model and in the two-dimensional side-plate model.

\section{APPENDIX II. DETERMINATION OF THE SLBSTITUTE LOAD FOR AN HOMEOSTATIC LOADING CYCLE}

As explained in the main text, it is assumed that the stimulus for bone adaptive activity in the cortex for a series of loads from a loading cycle can be expressed as

$$
S=B\left(\sum_{j=1}^{c} n_{j} U_{j}\right)^{1.4}
$$

where $B$ is a constant. For the series we take three loading cases suggested by Carter et al. (1987a), hence $c=3$, whereby $n_{1}=n_{2}=n_{3}=1$. The (schematized) geometrical configuration and the external loads are shown in Fig. A2.

The axial force within the borie cross-sections $N(x)$ can be found from

$$
N=F_{h} \cos x_{h}-F_{a} \cos x_{a s},
$$

and the bending moment $M(x)$ from

$M=F_{h}\left(h \cos x_{h}-x \sin x_{h}\right)+F_{a}\left(a \cos x_{a}+x \sin x_{a}\right)$. 
Table A1. Parameter values of the loading series

\begin{tabular}{llrrr}
\hline & & \multicolumn{3}{c}{ Value } \\
Parameter & Units & $j=1$ & $j=2$ & $j=3$ \\
\hline$F_{h}$ & $\mathrm{~N}$ & 2317 & 1158 & 1548 \\
$x_{h}$ & degrees & 24 & -15 & 56 \\
$h$ & $\mathrm{~mm}$ & 40 & 40 & 40 \\
$F_{a}$ & $\mathrm{~N}$ & 702 & 351 & 468 \\
$x_{a}$ & degrees & 28 & -8 & 35 \\
$a$ & $\mathrm{~mm}$ & 15 & 15 & 15 \\
$A$ & $\mathrm{~mm}^{2}$ & 392.7 & 392.7 & 392.7 \\
$I$ & $\mathrm{~mm}^{4}$ & $3.19 \times 10^{4}$ & $3.19 \times 10^{4}$ & $3.19 \times 10^{4}$ \\
\hline
\end{tabular}

Assuming the axisymmetric geometry of the bone, the maximal stress in the outer bone fibres $\sigma(x)$ follows from

$$
\sigma=\frac{N}{A}+\frac{M r_{a}}{I}
$$

where $A(x)$ and $I(x)$ are the area and moment of inertia, respectively. The SED can now be determined from

$$
U=\frac{\frac{1}{2} \sigma^{2}}{E} .
$$

Using estimates $a=15 \mathrm{~mm}$, and $h=40 \mathrm{~mm}$, the three loading cases suggested by Carter et al. (1987a), as specified in Table Al, and assuming the straight, axisymmetric geometry of the model discussed in the main text $\left(r_{\mathrm{b}}=15 \mathrm{~mm}\right.$, $r_{b}=10 \mathrm{~mm}$ ), the stimulus $S(x)$ was determined according to equation (AI) for $c=3$. The results, for the medial and the lateral sides, are presented in Fig. A2. Evidently, the stimulus pattern over the length of the proximal femoral diaphysis can be reasonably approximated by the stimulus of a pure bending moment $M$, also shown in Fig. A2.

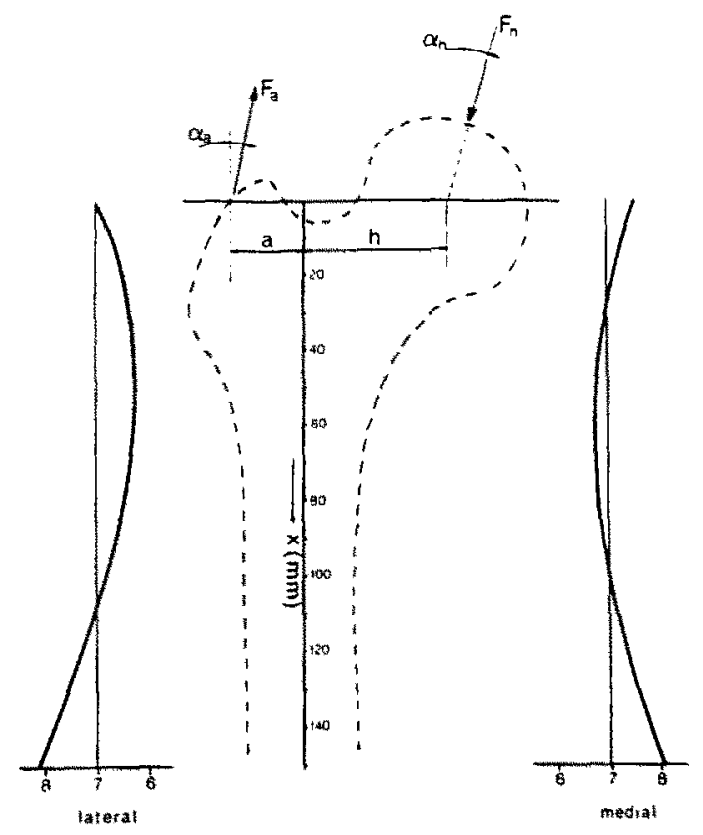

Fig. A2. Normalized distribution of the homeostatic stimulus $S$ in arbitrary units along the cortex of the bone at the medial and lateral sides, for the loading series 1 to 3 (compare Table A1). Also shown is the uniform distribution for a single substitute loading case, a pure bending moment $M$. 